\title{
Erratum to: No impact of severe varus deformity on clinical outcome after posterior stabilized total knee arthroplasty
}

\author{
Bum-Sik Lee $\cdot$ Sang-Jin Lee $\cdot$ Jong-Min Kim $\cdot$ \\ Dae-Hee Lee $\cdot$ Eun-Jong Cha $\cdot$ Seong-Il Bin
}

Published online: 11 January 2011

(C) Springer-Verlag 2010

\section{Erratum to: Knee Surg Sports Traumatol Arthrosc}

DOI 10.1007/s00167-010-1316-9

Unfortunately, we have given the information in Table 9 of our article incorrectly. Therefore, please find the corrected Table 9 here:

Table 9 The thicknesses of polyethylene inlays (mm)

\begin{tabular}{llll}
\hline & $\begin{array}{l}\text { Mild varus } \\
\text { group }\end{array}$ & $\begin{array}{l}\text { Severe varus } \\
\text { group }\end{array}$ & $P$ value \\
\hline Overall $^{\text {a }}$ & $13.9 \pm 2.5$ & $14.7 \pm 2.8$ & 0.049 \\
$\begin{array}{l}\text { Except of the augmentation } \\
\text { needed cases }\end{array}$ & $13.8 \pm 2.5$ & $15.1 \pm 2.7$ & 0.004 \\
\hline a The mean difference was $0.8 \mathrm{~mm}$ & & \\
b $\begin{array}{ll}\text { The mean difference was } 1.3 \mathrm{~mm} \text {, which was thicker than overall } \\
\text { comparison with more significant statistical difference }\end{array}$ &
\end{tabular}

The online version of the original article can be found under doi:10.1007/s00167-010-1316-9.

B.-S. Lee · S.-J. Lee · J.-M. Kim · S.-I. Bin $(\varangle)$

Department of Orthopaedic Surgery, Asan Medical Center,

College of Medicine, Ulsan University, 388-1, Pungnap-2-dong,

Songpa-gu, Seoul 138-736, South Korea

e-mail: sibin@amc.seoul.kr

D.-H. Lee

Department of Orthopaedic Surgery,

Korea University Anam Hospital, College of Medicine,

Korea University, Anam-dong 5-Ga, Seongbuk-gu,

Seoul 136-705, South Korea

\section{E.-J. Cha}

Department of Biomedical Engineering, College of Medicine, Chungbuk National University, Gaesin-dong, Heungdeok-gu,

Cheongju 361-763, Chungcheongbuk-do, South Korea 